UNIVERSIDADE DE SÃO PAULO

FACULDADE DE MEDICINA DE RIBEIRÃO PRETO

Carlos Alberto Ferreira Coelho Neto

Sinal Mismatch T2-FLAIR e sua relação com o prognóstico em gliomas difusos.

Ribeirão Preto

2020 
Carlos Alberto Ferreira Coelho Neto

\section{Sinal Mismatch T2-FLAIR e sua relação com o prognóstico em gliomas difusos.}

Dissertação apresentada à Faculdade de Medicina de Ribeirão Preto da Universidade de São Paulo, para obtenção do título de Mestre profissional pelo programa de mestrado profissional em Ciências das Imagens e Física Médica.

Área de Concentração: Diagnóstico por Imagem

Orientadores: Gustavo Novelino Simão

Antônio Carlos dos Santos

Versão corrigida.

A versão original encontra-se disponível tanto na Biblioteca da Unidade que aloja o Programa, quanto na Biblioteca Digital de Teses e Dissertações da USP (BDTD) 
Autorizo a reprodução e divulgação total ou parcial deste trabalho, por qualquer meio convencional ou eletrônico, para fins de estudo e pesquisa, desde que citada a fonte. 


\section{FICHA CATALOGRÁFICA}

Neto, Carlos Alberto Ferreira Coelho

Sinal Mismatch T2-FLAIR e sua relação com o prognóstico em gliomas difusos/ Carlos Alberto Ferreira Coelho Neto, Orientadores: Gustavo Novelino Simão, Antônio Carlos dos Santos.

- Ribeirão Preto, 2020.

35 páginas, 4 gráficos, 17 figuras.

Dissertação de mestrado profissional (Programa Ciências das Imagens e Física Médica Área de Concentração: Diagnóstico por Imagem) - Faculdade de Medicina de Ribeirão Preto da Universidade de São Paulo.

1) Ressonância magnética; 2) Gliomas difusos; 3) Mismatch T2-FLAIR 4) mutação IDH 5) codeleção 1p19q 6) prognóstico. 


\section{FOLHA DE APROVAÇÃO}

Carlos Alberto Ferreira Coelho Neto

Sinal Mismatch T2-FLAIR e sua relação com o prognóstico em gliomas difusos.

Dissertação apresentada à Faculdade de Medicina de Ribeirão Preto da Universidade de São Paulo para obtenção do título de mestreprofissionalizante.

Área de concentração: Diagnóstico por Imagem.

Aprovado em __ I I

Banca examinadora:

Prof. Dr

Instituicão:

Assinatura:

Prof. Dr

Instituicão:

Assinatura:

Prof. Dr

Instituicão:

Assinatura: 


\section{Agradecimentos}

Agradeço aos professores, colegas e amigos do Hospital das Clínicas da Faculdade de Medicina de Ribeirão Preto, da Universidade de São Paulo, pelos ensinamentos, apoio e amizade ao longo dos últimos anos.

Agradecimento ao apoio financeiro da CAPES. 


\section{RESUMO}

Coelho Neto, CAF. Sinal Mismatch T2-FLAIR e sua relação com o prognóstico em gliomas difusos. Dissertação de mestrado profissional. Faculdade de Medicina de Ribeirão Preto, Universidade de São Paulo.

Introdução: Os gliomas difusos de baixo grau (Grau 2 OMS) são tumores originados de tecido glial do sistema nervoso central, de crescimento habitualmente lento, com potencial de progressão para anaplasia (grau 3 OMS). Estes dois grupos são caracterizados como gliomas de menor grau neste trabalho (1). Atualmente, em adição ao estudo histológico habitual, a análise molecular vem se consolidando como principal ferramenta diagnóstica nestes casos, visto que diferentes subtipos tumorais apresentam comportamentos biológicos diferentes, com impacto no tratamento e sobrevida dos pacientes. Há a recente descrição de um marcador de imagem em ressonância magnética, denominado Mismatch T2-FLAIR, que apresenta correlação com um subtipo molecular específico de glioma difuso.

Objetivo: Identificar, entre os pacientes com gliomas difusos, a presença do sinal Mismatch T2-FLAIR no exame de ressonância magnética e se existe correlação com o prognóstico nestes casos.

Método: foram incluídos e avaliados casos de gliomas difusos do sistema nervoso central (Grau II e III OMS) dos últimos 15 anos. O aspecto de imagem na ressonância magnética foi avaliado, em busca do sinal Mismatch T2-FLAIR. Posteriormente, foram obtidos dados prognósticos de todos os pacientes do estudo, no intuito de comparar a sobrevida global e a sobrevida em relação a progressão de doença dos pacientes portadores ou não do sinal em questão. A sobrevida também foi avaliada em relação a outras variáveis isoladas, com idade, sexo, tipo histológico e grau tumoral.

Resultados: não houve diferença estatisticamente significatica na sobrevida, tanto em relação a óbito quanto a progressão de doença, entre os pacientes portadores ou não do sinal Mismatch T2-FLAIR. Na análise das outras variáveis isoladas, houve sobrevida significativamente inferior dos pacientes com tumores grau III em relação aos de grau II (OMS).

Conclusão: Como fator isolado de prognóstico, nosso estudo não demonstrou relação do sinal Mismatch T2-FLAIR com o prognóstico nos pacientes portadores deste grupo de tumor. 
Palavras-chave: ressonância magnética; gliomas difusos; mismatch T2-FLAIR; mutação IDH; codeleção 1p19q. 


\section{ABSTRACT}

Coelho Neto, CAF, The prognostic value of T2-FLAIR Mismatch Sign in diffuse gliomas. 2020. Master's degree (or thesis). Ribeirão Preto Medical School, University of São Paulo.

Introduction: Low grade gliomas (OMS grade II) are originated from glial tissue in central nervous system, habitually with slow growth and with possibility of progression to anaplasia (WHO grade III). These two groups are called lower grade gliomas in this study. Nowadays, in addition to habitual histologic analysis, the mollecular pattern is becoming the main diagnostic tool in these cases, as different tumor subtypes have different biological behaviours, with impact in therapy and survival rates. In this context, there has been recently described an image marker in magnetic resonance imaging, called Mismatch T2-FLAIR, which correlates to a specific molecular subtype of diffuse glioma.

Objectives: Identify, among patients with diffuse gliomas, the presence of Mismatch T2-FLAIR sign in magnetic resonance imaging. Then, compare the survival rates between these patients and the ones without the image sign.

Methods: we included and evaluated diffuse glioma cases (WHO grade II AND III) treated in the last 15 years in our institution. The MRI was evaluated searching for the Mismatch T2-FLAIR sign. Then, we searched for prognostic data of all patients, in order to compare global and disease progression survival rates between the patients with presence or absence of the image sign. The survival rates were also evaluated considering other variants, such as histologic type, sex, tumor grade and age.

Results: there was no statistically significant difference in survival rates between patients with diffuse gliomas and presence or absence of the Mismatch T2FLAIR sign, considering obit or even disease progression. The analysis of other variants showed significant lower survival rates in patients with WHO grade III tumors than in WHO II tumors.

Conclusion: As an isolated prognostic marker, our study did not show relation between the image sign and prognostic rates in patients with diffuse gliomas.

Key words: magnetic resonance; diffuse gliomas; Mismatch T2/FLAIR; IDH mutation, $1 \mathrm{p} 19 \mathrm{q}$ codeletion. 


\section{Lista de abreviaturas e siglas}

- OMS: Organização Mundial de Saúde

- GMG: gliomas de menor grau

- IDH: isocitrato desidrogenase

- RM: ressonância magnética

- USP: Universidade de São Paulo

- HCFMRP: Hospital das Clínicas da Faculdade de Medicina de Ribeirão Preto

- FLAIR: fluid-attenuated inversion recovery

- CEP: comitê de ética e pesquisa. 


\section{Lista de gráficos}

- Gráfico 1: Distribuição da amostra em relação presença do sinal Mismatch T2Flair na ressonância magnética.

- Gráfico 2. Distribuição da amostra em relação ao sexo.

- Gráfico 3: Distribuição da amostra em relação ao tipo histológico.

- Gráfico 4: Distribuição da amostra em relação ao grau tumoral. 


\section{Lista de figuras}

- Figura 1: Classificações histopatológicas e moleculares dos gliomas difusos

- Figura 2: Proposta de diagnóstico em camadas em tumores do sistema nervoso central.

- Figuras 3, 4, 5: Sinal Mismatch T2-FLAIR na ressonância magnética.

- Figura 6: ausência do Sinal Mismatch T2-FLAIR.

- Figura 7: Estimativa de sobrevida global pelo método Kaplan Meyer em relação ao sinal Mismatch T2/FLAIR.

- Figura 8: Estimativa de sobrevida global pelo método Kaplan Meyer em relação ao sexo.

- Figura 9: Estimativa de sobrevida global pelo método Kaplan Meyer em relação ao tipo histológico.

- Figura 10: Estimativa de sobrevida global pelo método Kaplan Meyer em relação ao grau tumoral.

- Figura 11: Estimativa de sobrevida global pelo método Kaplan Meyer em relação a idade.

- Figura 12: Estimativa de sobrevida (progressão de doença) pelo método Kaplan Meyer em relação ao sinal Mismatch T2/FLAIR.

- Figura 13: Estimativa de sobrevida (progressão de doença) pelo método Kaplan Meyer em relação ao sexo.

- Figura 14: Estimativa de sobrevida (progressão de doença) pelo método Kaplan Meyer em relação ao tipo histológico.

- Figura 15: Estimativa de sobrevida (progressão de doença) pelo método Kaplan Meyer em relação ao grau tumoral.

- Figura 16: Estimativa de sobrevida (progressão de doença) pelo método Kaplan Meyer em relação a idade. 


\section{SUMÁRIO}

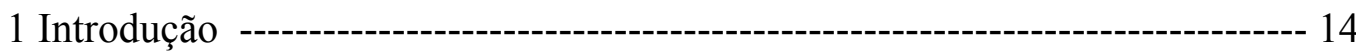

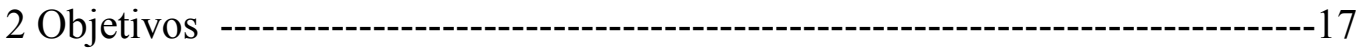

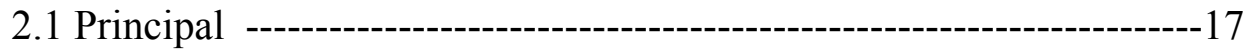

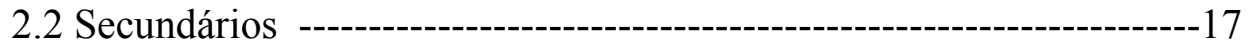

3 Material e métodos ---

3.1 Tipo de estudo -----

3.2 Local do estudo ----o-

3.3 Comitê de ética ---

3.4 Coleta de dados -----------------------------------------------------18

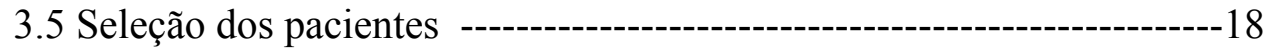

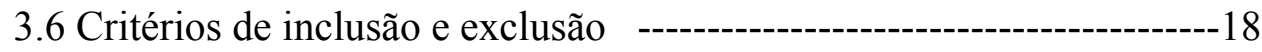

3.6.1 Critérios de inclusão ---

3.6.2 Critérios de exclusão ---

3.7 Análise das imagens ---------------------------------------------19

3.7.1 Sinal Mismatch T2-FLAIR ---------------------------19

3.8 Dados demográficos, clínicos e patológicos ------------------------21

3.8.1 Desfechos -----o---21

3.8.1.1 Sobrevida global ----o--o---21

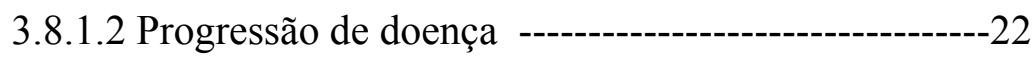

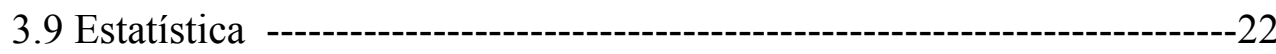

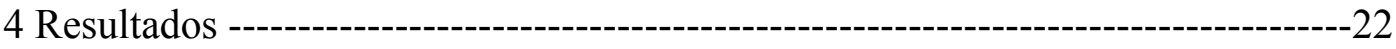

4.1 Sobrevida para óbito ou último registro do paciente com vida ---------23

4.1.1 Presença x ausência do sinal Mismatch T2/FLAIR ----------------23

4.1.2 Sexo masculino x feminino -----------------------------------23

4.1.3 Astrocitomas $\mathrm{x}$ oligodendrogliomas $\mathrm{x}$ oligoastrocitomas ----------24

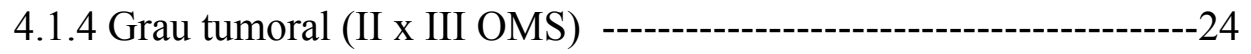

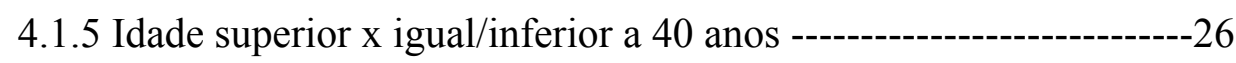

4.2 Sobrevida para progressão de doença ou última vez visto ---------------26

4.2.1 Presença x ausência do sinal Mismatch T2/FLAIR --------------- 27

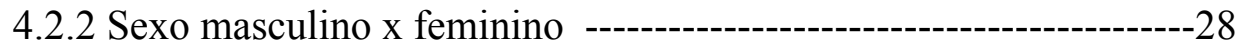

4.2.3 Astrocitomas $\mathrm{x}$ oligodendrogliomas $\mathrm{x}$ oligoastrocitomas ----------28

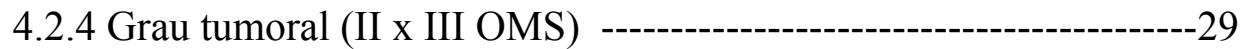

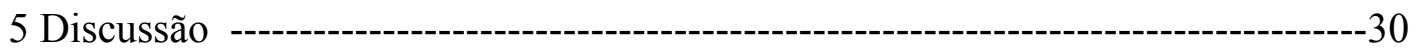

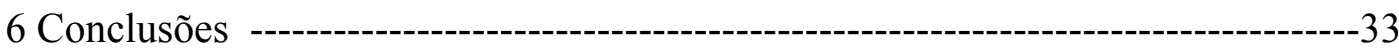

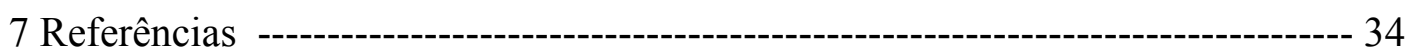




\section{1 - INTRODUÇÃO}

Os gliomas são tumores originados de tecido glial do sistema nervoso central, sendo os tumores primários cerebrais mais comuns no adulto. São incluídos neste grupo os tipos histológicos dos astrocitomas e oligodendrogliomas, bem como glioblastomas, estes últimos os mais freqüentes neste espectro.

Os gliomas difusos de baixo grau (Grau 2 Organização Mundial da Saúde [OMS]) têm crescimento habitualmente lento, com potencial de progressão para anaplasia (grau 3 OMS). Estes dois grupos são caracterizados como gliomas de menor grau (GMG) neste trabalho (1).

Trata-se de um conjunto de neoplasias cerebrais infiltrativas e tradicionalmente eram classificados com base histológica, tendo como parâmetro a célula de origem, em astrocitomas, oligodendrogliomas e oligoastrocitomas (2). Estes tumores eram então agrupados em graus II ou III pela Organização Mundial de Saúde - OMS baseado em características morfológicas que refletiam comportamento biológico, como mitoses, proliferação vascular e necrose, podendo ainda ser classificados em grau IV (OMS), neste caso caracterizando um glioblastoma $(2,3,4)$.

A classificação histológica dos gliomas em geral é sujeita a erros de amostra durante o biópsia/ressecção, e, mais importante, sofre de alta variabilidade intra e interobservador $(1,2,5)$. Além disso, sabe-se que existem diferentes características de evolução, resposta a tratamento, e sobrevida entre tumores com o mesmo fenótipo, o que reforça a importância do diagnóstico acurado (8).

Neste contexto, características moleculares deste grupo de tumores vêm sendo estudadas, contribuindo para seu maior entendimento, bem como para sua classificação e associação com sobrevida $(5,6)$. A evolução nos parâmetros moleculares levou à inclusão de novas características envolvendo os gliomas difusos na Classificação de Tumores do Sistema Nervoso Central da OMS - 2016, em particular as mutações na isocitrato desidrogenase 1 e 2 (em conjunto referidas como IDH) e codeleção dos cromossomos $1 \mathrm{p}$ e $19 \mathrm{q} .(3,5,7)$

Nesta nova classificação (Figura 1), os gliomas difusos podem ser agrupados em três classes: 1) Gliomas de menor grau (GMG - II e III) com mutação IDH e com codeleção 1p/19q, que direcionam o diagnóstico para o grupo dos oligodendrogliomas; 2) GMG com mutação IDH e sem codeleção 1p/19q, que direcionam para o grupo dos astrocitomas; 3) GMG sem mutação IDH (IDH selvagem) e sem codeleção 1p/19q, estes com comportamento semelhante aos glioblastomas (Grau IV) $(3,5)$. Os três grupos 
apresentam evoluções e sobrevida diferentes, e, sendo a classificação genética mais acurada que a histológica, existe a tendência a confiar neste parâmetro para guiar a decisão clínica (9). Por exemplo, o grupo 1), IDH mutado e com codeleção 1p/19q, apresenta melhor resposta ao tratamento radio e quimioterápico e apresenta maior sobrevida que os grupos sem este perfil $(8,9)$.

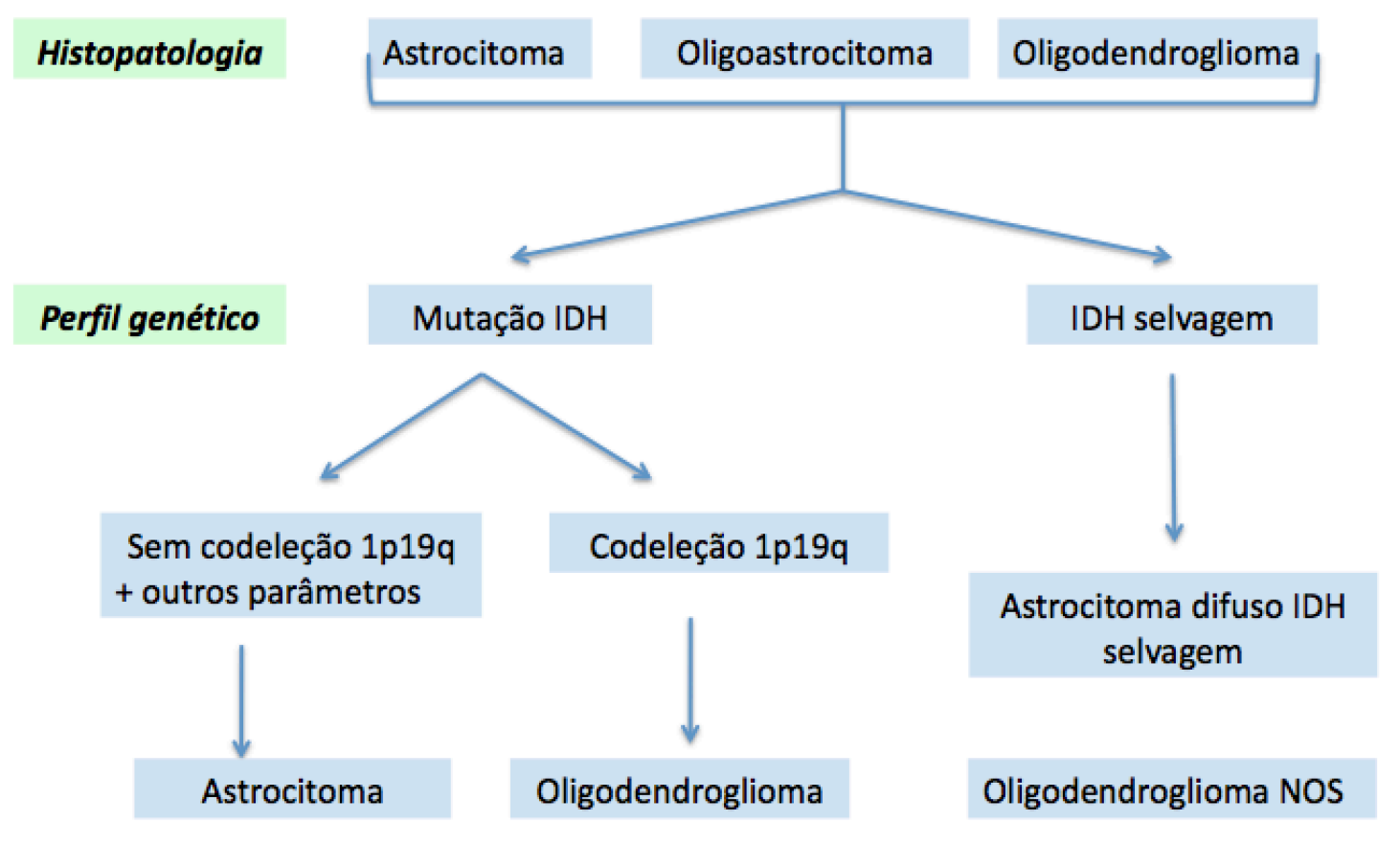

Figura 1: Classificações histopatológicas e moleculares dos tumores do sistema nervoso central.

Adaptado de: The 2016 World Health Organization Classification of Tumors of the Central Nervous System: a summary

Torna-se necessário, portanto, considerar todas as informações disponíveis para o diagnóstico mais preciso dos gliomas difusos, o que levou a ser estabelecido o conceito do diagnóstico em camadas, que considera os aspectos histopatológico, grau tumoral e perfil molecular para o diagnóstico final (Figura 2).

\section{Nível 1 Diagnóstico final integrado \\ Nível 2 Classificação histológica \\ Nível 3 Grau OMS \\ Nível 4 Perfil molecular}

Figura 2: Proposta de diagnóstico em camadas em tumores do sistema nervoso central.

Habitualmente, os gliomas difusos apresentam-se, na ressonância magnética, como lesões expansivas predominantemente lobares, com limites parcialmente 
delimitados, hipointensas nas sequências ponderadas em $\mathrm{T} 1$ e hiperintensas em T2/FLAIR. Podem apresentar focos de calcificações e o realce pelo meio de contraste é variável, mais freqüente nos casos de anaplasia.

A diferenciação entre os tipos histológicos de astrocitomas e oligodendrogliomas não é possível apenas por critérios de imagem. Os tumores apresentam características semelhantes e sobreponíveis, sendo possível, em alguns casos, suspeitar de um determinado tipo histológico diante de algumas algumas diferenças bem estabelecidas no exame de imagem. Entre elas, as mais freqüentes são a presença de calcificações, representadas por focos de hipossinal nas sequências de susceptibilidade magnética e mais freqüentes nos oligodendrogliomas; e o predomínio de acometimento subcortical nos casos de astrocitomas, versus envolvimento predominantemente corticossubcortical nos casos de oligodendrogliomas (14).

Em relação a epidemiologia, os gliomas difusos de baixo grau apresentam características em comum, considerando que os oligodendrogliomas têm pico entre 40 e 45 anos, com moderada predominância no sexo masculino, enquanto os astrocitomas supratentoriais de baixo grau apresenta predomínio entre 20 e 45 anos, também predominando em pacientes do sexo masculino (14).

Recentemente, os estudos de Patel et al (5), com resultados semelhantes no estudo de validação de Broen et al (7), demonstraram um possível marcador de imagem em ressonância magnética para o subgrupo molecular IDH mutado sem codeleção 1p/19q (Grupo 2, que engloba astrocitoma graus II e III), denominado sinal Mismatch T2-FLAIR, que demonstrou valor preditivo positivo de $100 \%$ para este grupo $(5,8)$.

O sinal é caracterizado por hipersinal da lesão na sequência ponderada em T2, em combinação com hipossinal relativo na sequência FLAIR (fluid attenuation inversion recovery), exceto por halo hiperintenso periférico (5).

Ao contribuir para a distinção entre diferentes subgrupos da classificação da OMS 2016, o sinal de imagem pode contribuir no diagnóstico e manejo clínico precoce do paciente com diagnóstico de glioma difuso. O tratamento dos gliomas difusos envolve modalidades terapêuticas como ressecção cirúrgica, radioterapia e quimioterapia. O envolvimento de áreas eloquentes pela lesão tumoral é um dos fatores considerados na programação terapêutica, e ter um dado prognóstico fornecido pelo exame de imagem antes da biópsia/ressecção cirúrgica pode ser de grande valor nesta avaliação(5,8). 


\section{2 - OBJETIVOS}

\subsection{Principal:}

Comparar o prognóstico entre os pacientes com diagnóstico histopatológico de gliomas difusos com presença ou ausência do sinal Mismatch T2/FLAIR na ressonância magnética. A presença do sinal é um marcador isolado de melhor ou pior prognóstico?

\subsection{Secundários:}

Comparar o prognóstico entre os pacientes com diagnóstico histopatológico de gliomas difusos envolvendo outras variáveis, como sexo, idade, tipo histológico e grau tumoral. 


\section{3 - MATERIAL E MÉTODOS}

\subsection{Tipo de estudo}

Trata-se de um estudo retrospectivo e observacional, realizado em uma única instituição.

\subsection{Local do estudo}

O estudo foi realizado no hospital das Clínicas da Faculdade de Medicina de Ribeirão Preto da Universidade de São Paulo (HCFMRP-USP).

\subsection{Comitê de ética}

Este projeto segue a resolução CNS 466/12 e foi encaminhado para apreciação do Comitê de Ética em Pesquisa (CEP) do Hospital das Clínicas da Faculdade de Medicina de Ribeirão Preto (HCFMRP-USP). Não houve deste modo, coleta de dados antes da aprovação pelo CEP. Também foi encaminhado ao CEP documento em anexo, solicitando a dispensa da necessidade do termo de consentimento, visto que se trata de estudo retrospectivo e o presente trabalho não alterará, de forma alguma, a conduta já tomada frente ao quadro do paciente. Os dados foram obtidos através de exames prévios realizados pelos pacientes. Ressaltamos ainda, que foram tomadas medidas para que se preserve o anonimato absoluto das informações dos pacientes.

\subsection{Coleta de dados:}

Os dados clínicos e histopatológicos foram colhidos a partir do Sistema de Informações Hospitalares. As imagens de ressonância magnética foram obtidas a partir do PACS (Picture and Archiving System) do Centro de Ciências das Imagens e Física Médica (CCIFM), onde estão armazenadas.

\subsection{Seleção dos pacientes:}

Inicialmente, foram identificados pacientes operados pela equipe de Neurocirurgia do HCFMRP-USP, com diagnóstico histopatológico de gliomas, entre os 
anos de 2003 a 2019, e que mantiveram período de acompanhamento ambulatorial pósoperatório nesta instituição.

\subsection{Critérios de inclusão e exclusão:}

Foram excluídos pacientes com diagnóstico histopatológico de glioblastoma (OMS grau IV), pelo fato de que o sinal em estudo não se aplica nestes casos, bem como pacientes operados após 2017, por apresentarem tempo de seguimento inferior a 2 anos até o início do trabalho.

Foram então incluídos os pacientes com diagnóstico histopatológico de gliomas difuso (OMS graus II e III), incluindo astrocitomas, oligodendrogliomas e oligoastrocitomas, entre os anos de 2000 a 2017, totalizando 97 pacientes. Destaca-se que os diagnósticos de oligoastrocitomas foram atribuídos antes da última atualização da OMS 2016.

\subsubsection{Critérios de inclusão:}

a) Pacientes operados com diagnóstico histopatológico de gliomas difusos (OMS graus II e III) entre os anos de 2000 a 2017.

b) Possuir um exame de imagem de ressonância magnética de encéfalo pré-operatória realizada no HCFMRP-USP.

\subsubsection{Critérios de exclusão:}

a) Pacientes com diagnóstico histopatológico de glioblastomas (OMS IV).

b) Pacientes sem exame pré-operatório de ressonância magnética disponível.

c) Pacientes operados com diagnóstico realizado posteriormente a 2017.

\subsection{Análise das imagens}

Os exames de ressonância magnética foram realizados em aparelho Phillips de 3,0T.

As imagens de RM foram analisadas por um médico radiologista, durante especialização na área de neurorradiologia, em busca do sinal Mismatch T2/FLAIR, 
utilizando o software visualizador Horos, versão 1.1.7. Em casos de dúvidas acerca da presença ou ausência do sinal em questão, o exame foi avaliado por neurorradiologista com mais de 10 de anos de experiência e um consenso foi então obtido.

\subsubsection{Sinal Mismatch T2/FLAIR:}

A análise qualitativa dos exames de ressonância magnética em busca sinal do sinal Mismatch T2/FLAIR foi realizada nas sequências T2 e FLAIR. O sinal foi considerado presente quando caracterizado hipersinal na sequência T2 e hipossinal na sequência FLAIR, exceto por halo hiperintenso nesta última sequência (Figura 3). 

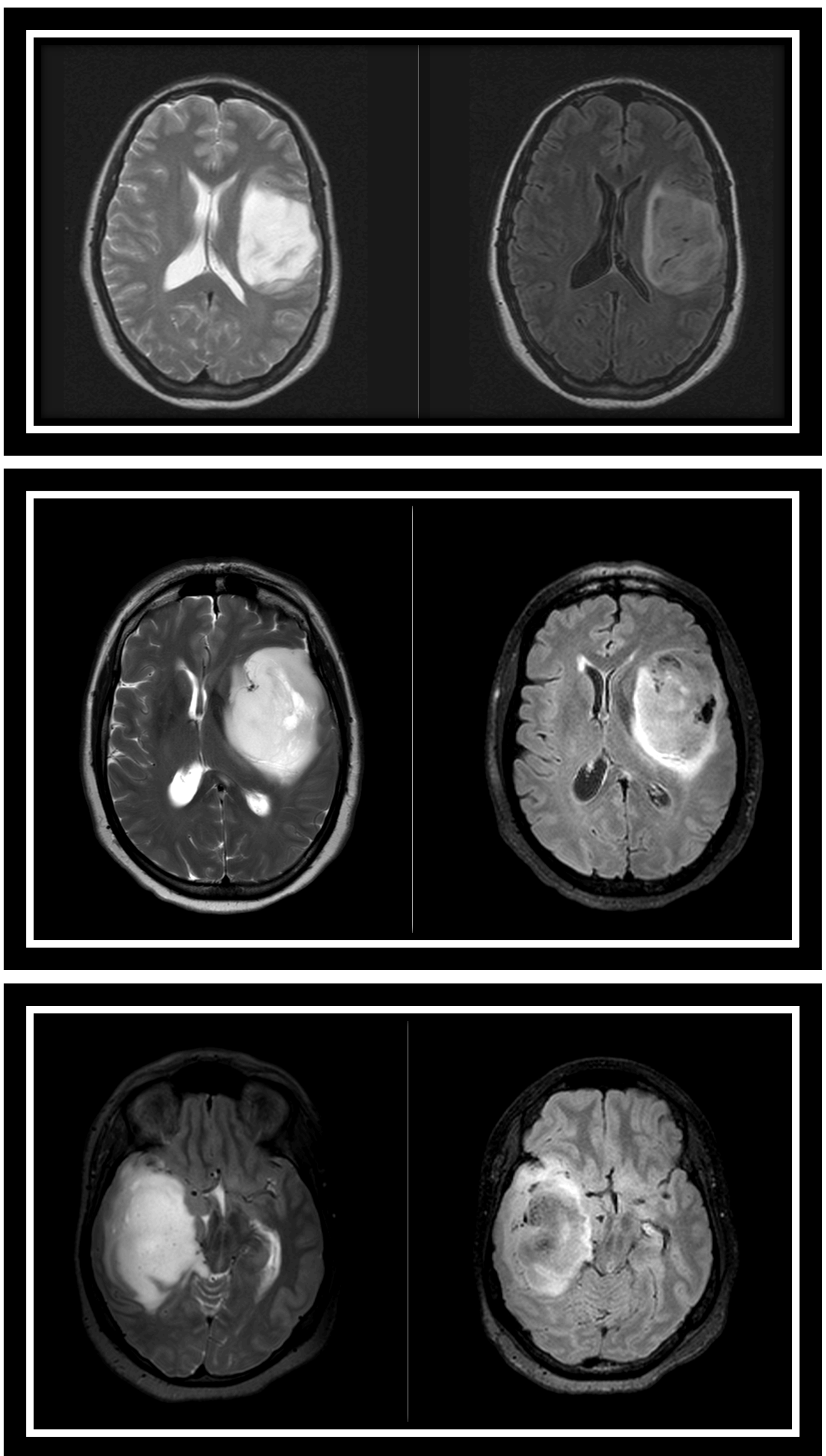

Figura 3: sequências T2 (esquerda) e FLAIR (direita) de ressonância magnética de três pacientes portadores de gliomas difusos com 


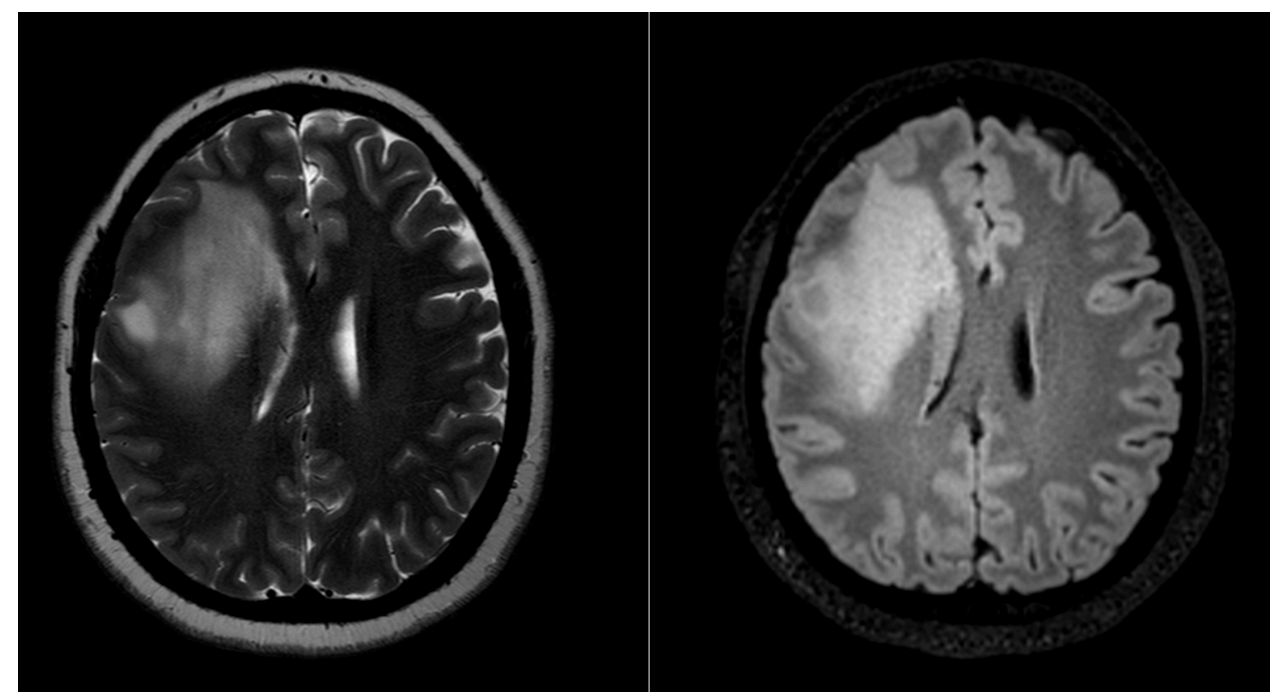

Figura 4: sequências T2 (esquerda) e FLAIR (direita) de ressonância magnética de paciente portador de glioma difuso com confirmação histopatológica e ausência do sinal Mismatch T2- FLAIR.

\subsection{Dados demográficos, clínicos e patológicos:}

Os parâmetros clínicos e as informações acerca da evolução dos pacientes foram coletados por um radiologista através do prontuário eletrônico da instituição.

Foram analisados e coletados os dados: (1) idade, (2) sexo, (3) tipo histopatológico (4) grau tumoral - OMS, (5) tempo de seguimento, progressão de doença e óbito.

O tempo de seguimento foi detalhado em meses, sendo cronometrado desde a data do primeiro exame de imagem até o ultimo registro vivo do paciente na instituição ou data de óbito.

A progressão de doença foi caracterizada através de critérios de imagem nos exames de ressonância magnética realizados no seguimento pós-operatório.

\subsubsection{Desfechos:}

\subsubsection{Em relação a sobrevida global:}

$\mathrm{Na}$ análise de sobrevida global, independentemente de progressão da doença, foram considerados, como desfechos, óbito ou última data de seguimento clínico. 


\subsubsection{Em relação a progressão de doença:}

$\mathrm{Na}$ análise de sobrevida em relação a progressão doença, foram considerados como desfechos a progressão de doença por critérios de imagem de ressonância magnética ou última data de seguimento do paciente sem relato de progressão.

\subsection{Estatística:}

A estimativa de sobrevida foi realizada pelo método de Kaplan-Meyer e as comparações foram realizadas pelo teste de Log Rank. Para todas as análises estatísticas foi considerado o nível de significância de 0,05 . 


\section{4 - RESULTADOS}

Foram avaliados 97 pacientes com diagnóstico histopatológico de GMG quanto a presença ou ausência do sinal Mismatch T2/FLAIR.

Dos 97 pacientes avaliados, 21 apresentaram o sinal em estudo $(21,6 \%)$, sendo $59(60 \%)$ do sexo masculino e 38 (40\%) do sexo feminino. Em relação aos tipos histológicos, 49 (50,5\%) tinham o diagnóstico histopatológico de oligodendrogliomas, $22(22,6 \%)$ de astrocitomas e $26(26,8 \%)$ de oligoastrocitomas. 65 (67\%) dos tumores foram categorizados como grau II e 32 (33\%) como grau III (OMS).

A idade média dos pacientes com o sinal Mismatch T2-FLAIR na ressonância magnética foi de 36,8 anos (desvio padrão [DP] de 8,8 anos) e de 41 anos (DP: 11,0 anos) em pacientes sem o sinal. A idade média geral da amostra foi de 40,1 anos (DP: 10,6 anos).

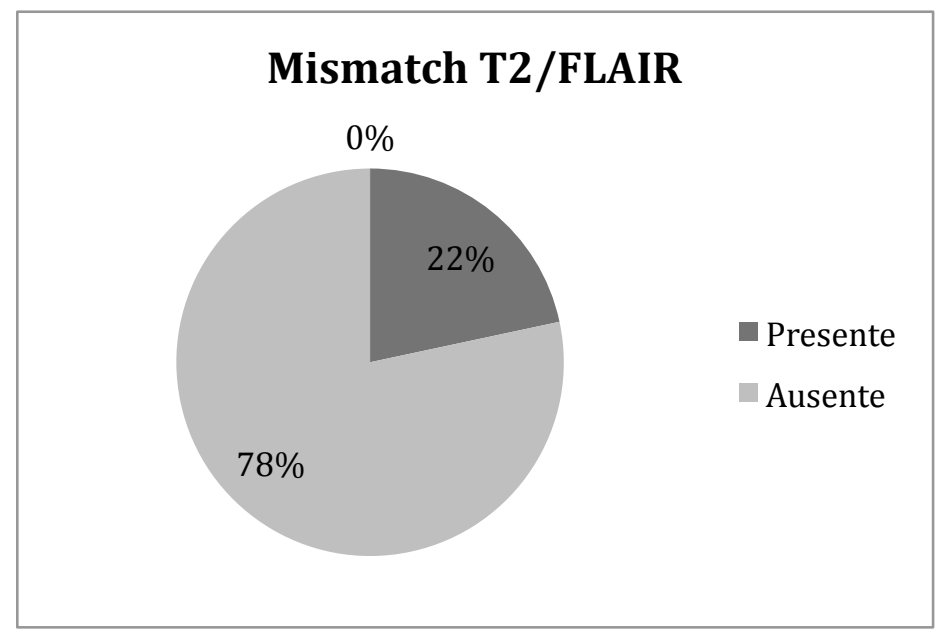

Gráfico 1: Distribuição da presença do sinal Mismatch T2-Flair 


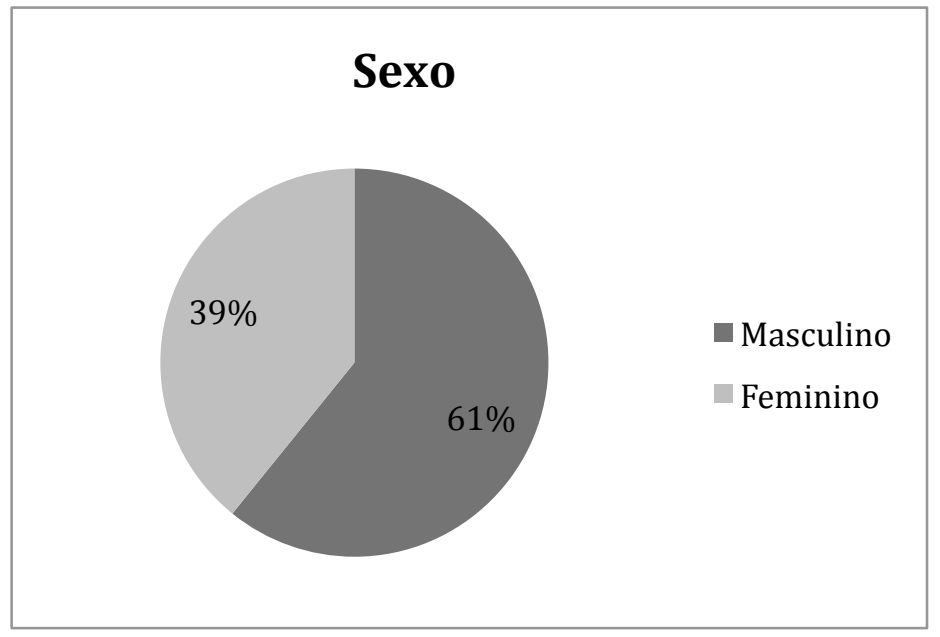

Gráfico 2: Distribuição em relação ao sexo

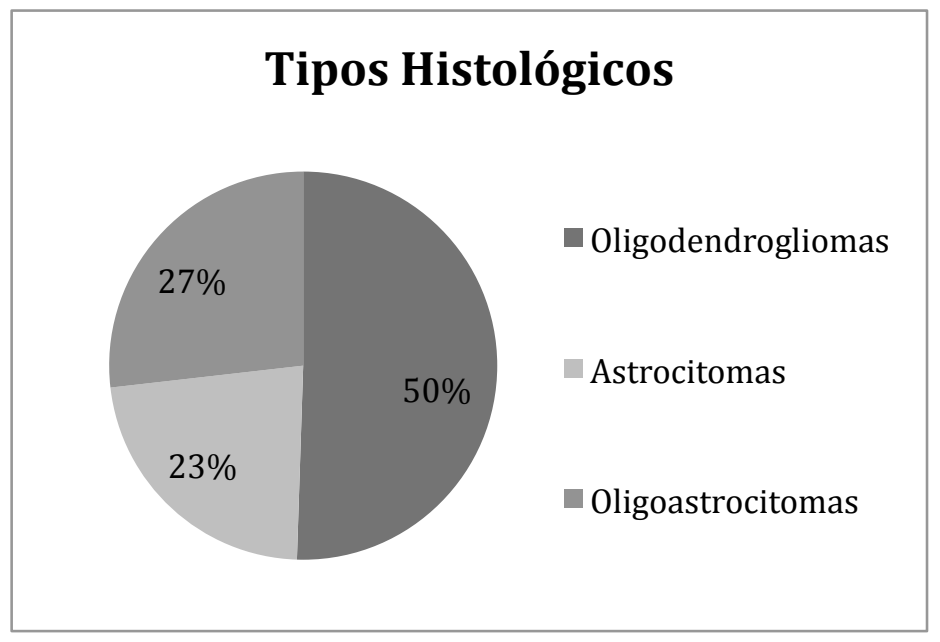

Gráfico 3: Distribuição em relação ao tipo histológico

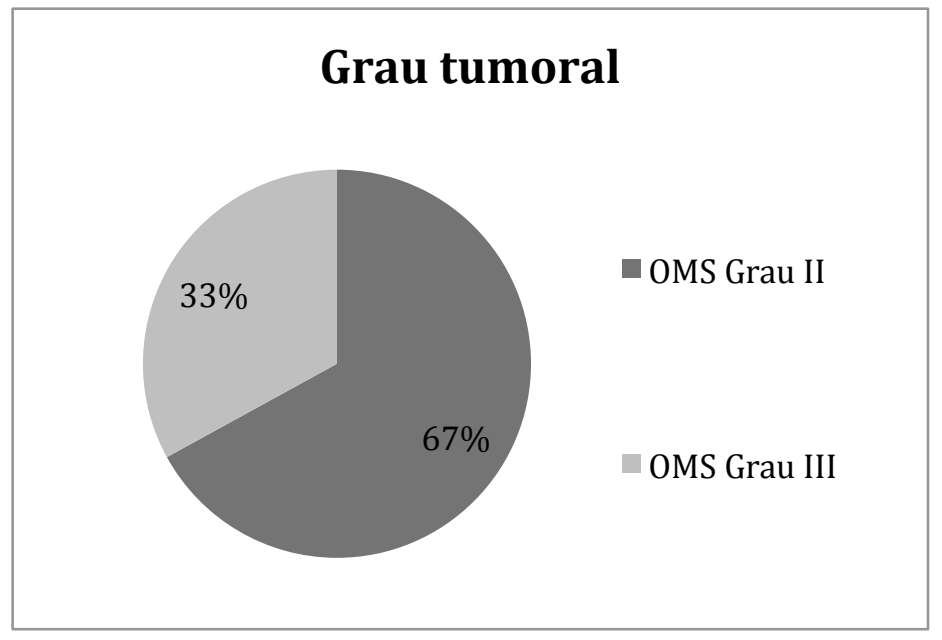

Gráfico 4. Distribuição em relação ao grau tumoral (OMS) 
4.1. Análise e comparação da sobrevida considerando os desfechos de óbito ou último registro do paciente com vida, independentemente da presença ou ausência de progressão tumoral:

4.1.1. Entre os grupos com presença e ausência do sinal Mismatch T2/FLAIR:

A análise estatística demonstrou não haver diferença significativa na sobrevida entre os grupos com presença e ausência do sinal Mismatch T2/FLAIR ( $\mathrm{p}=$ $0,62)$.

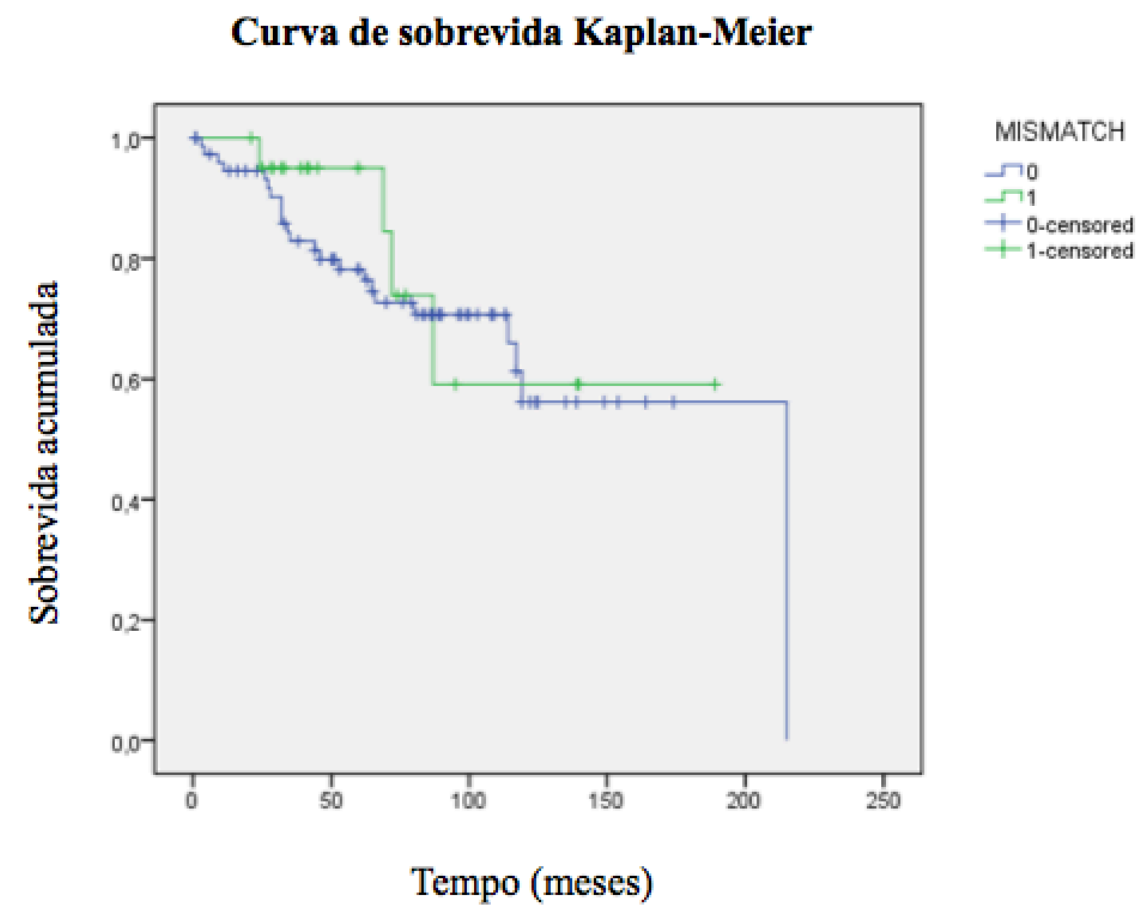

Figura 7: Estimativa de sobrevida pelo método Kaplan Meyer. Em azul: ausência do sinal Mismatch T2/FLAIR. Em verde: presença do sinal.

4.1.2 Comparação da sobrevida entre os grupos de sexo masculino e feminino:

A análise estatística demonstrou não haver diferença significativa na sobrevida entre os sexos $(p=0,24)$. 


\section{Curva de sobrevida Kaplan-Meier}

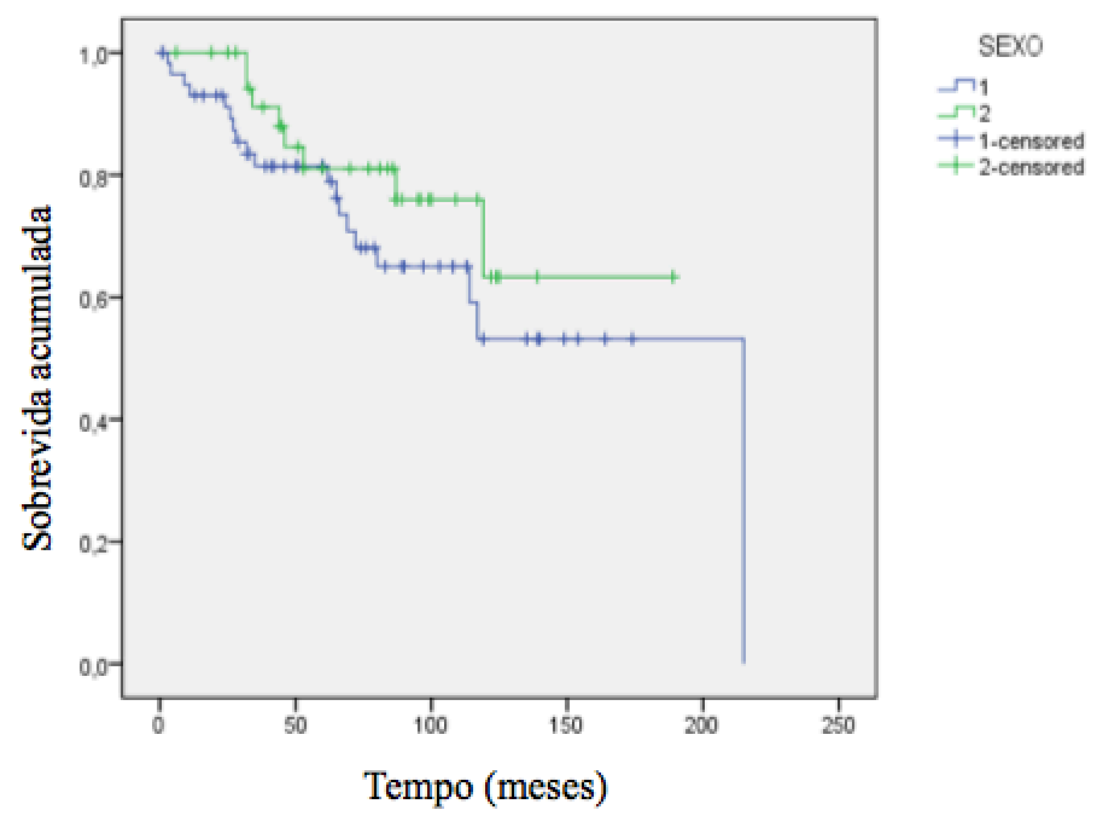

Figura 8: Estimativa de sobrevida pelo método Kaplan Meyer. Em azul: sexo masculino. Em verde: sexo feminino.

\subsubsection{Entre os três tipos histopatológicos (astrocitomas,} oligodendrogliomas e oligoastrocitomas):

A análise estatística demonstrou não haver diferença significativa na sobrevida entre os diferentes tipos histológicos $(p=0,24)$.

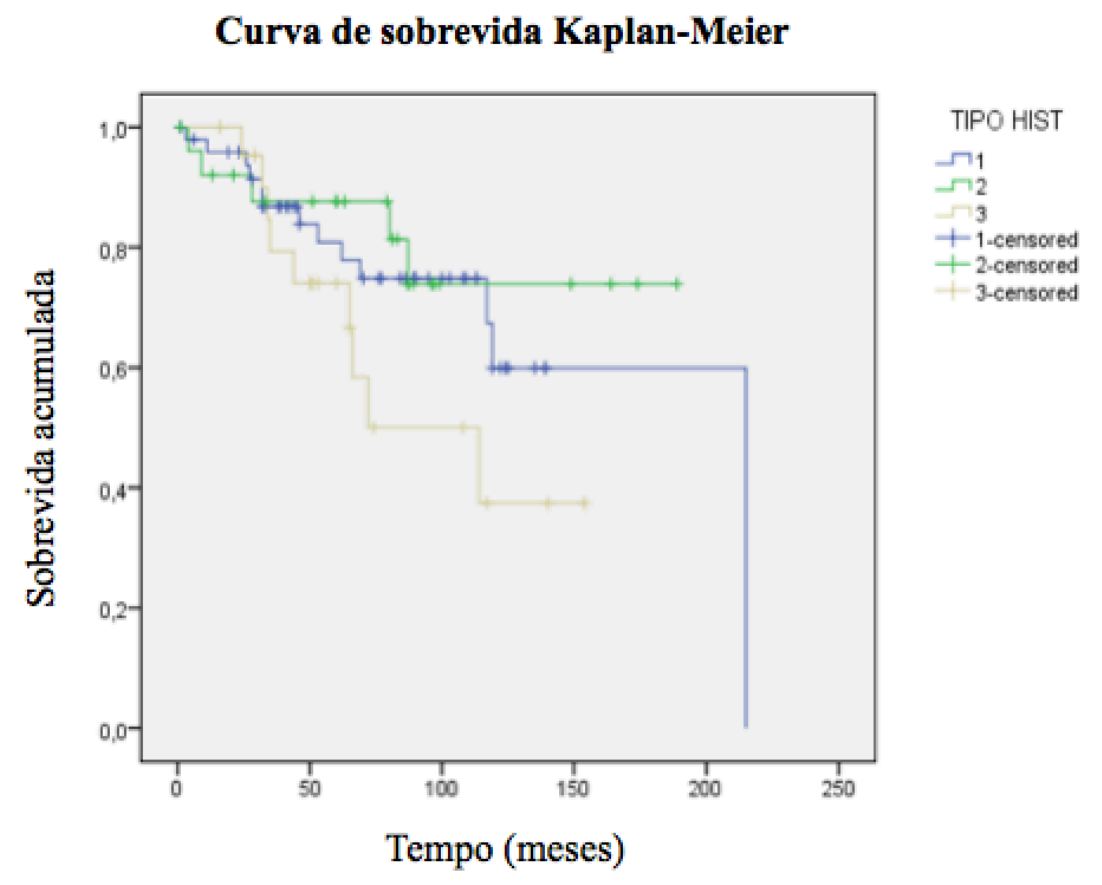

Figura 9: Estimativa de sobrevida pelo método Kaplan Meyer. Em azul: oligodendrogliomas. Em verde: oligoastrocitomas. Em amarelo: astrocitomas. 
4.1.4 Comparação da sobrevida entre os diferentes graus tumorais (II III) segundo a OMS 2016:

A análise estatística demonstrou haver diferença significativa na sobrevida entre os graus tumorais, com sobrevida estatisticamente superior no grupo de tumores grau II OMS $(\mathrm{p}=0,025)$.

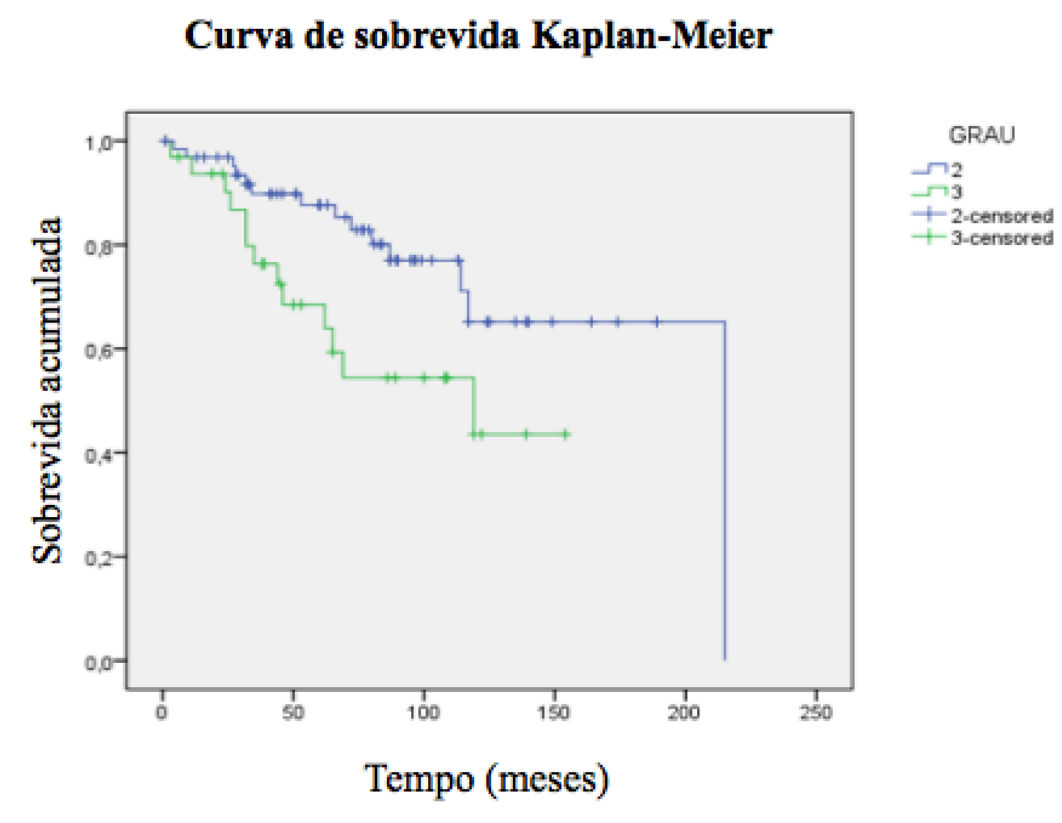

Figura 10: Estimativa de sobrevida pelo método Kaplan Meyer. Em azul: gliomas difusos grau II. Em verde: grau III.

\subsubsection{Entre os pacientes com idade superior ou igual/inferior a 40 anos} no momento do diagnóstico:

A análise estatística demonstrou demonstrando não haver diferença significativa na sobrevida entre os grupos de idade superior ou igual/inferior a 40 anos $(\mathrm{p}=0,31)$ 


\section{Curva de sobrevida Kaplan-Meier}

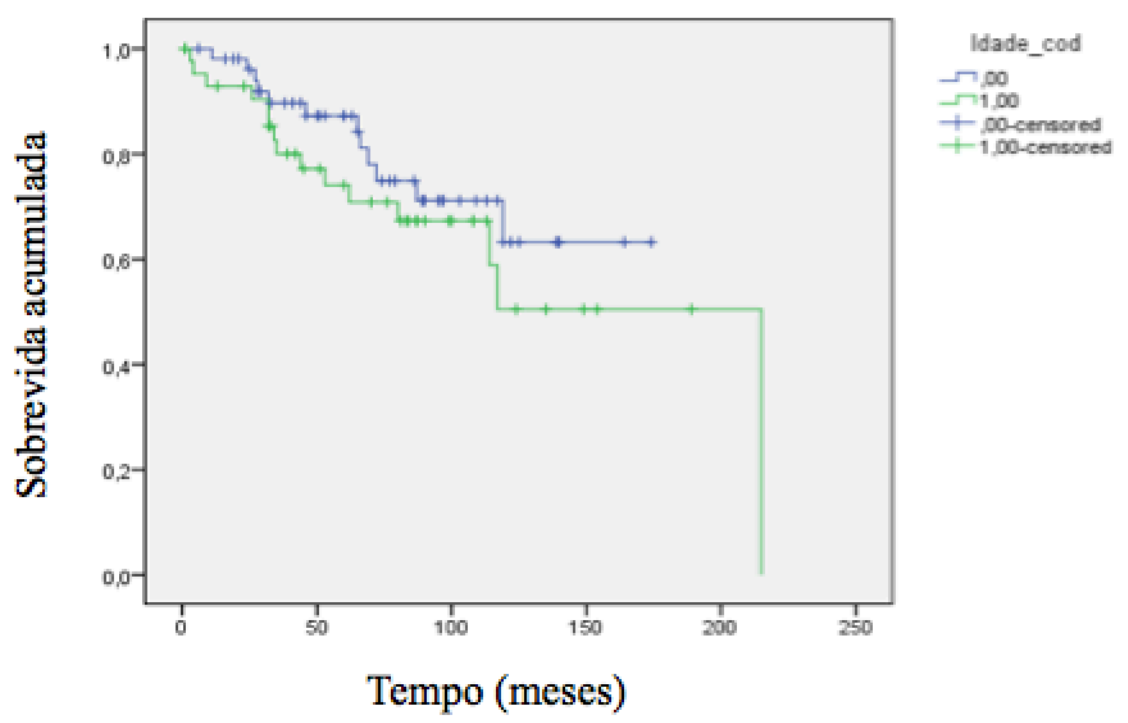

Figura 11: Estimativa de sobrevida pelo método Kaplan Meyer. Em azul: grupo com idade inferior a 40 anos. Em verde: idade superior a 40 anos.

4.2 Análises de sobrevida considerando como desfechos progressão de doença após a primeira cirurgia ou última vez visto sem progressão.

4.2.1 Entre os grupos com presença ou ausência do sinal Mismatch T2/FLAIR em relação a progressão de doença:

A análise estatística demonstrou não haver diferença na sobrevida em relação a progressão de doença nos grupos com presença ou ausência do sinal Mismatch T2/FLAIR $(\mathrm{p}=0,80)$. 


\section{Curva de sobrevida Kaplan-Meier}

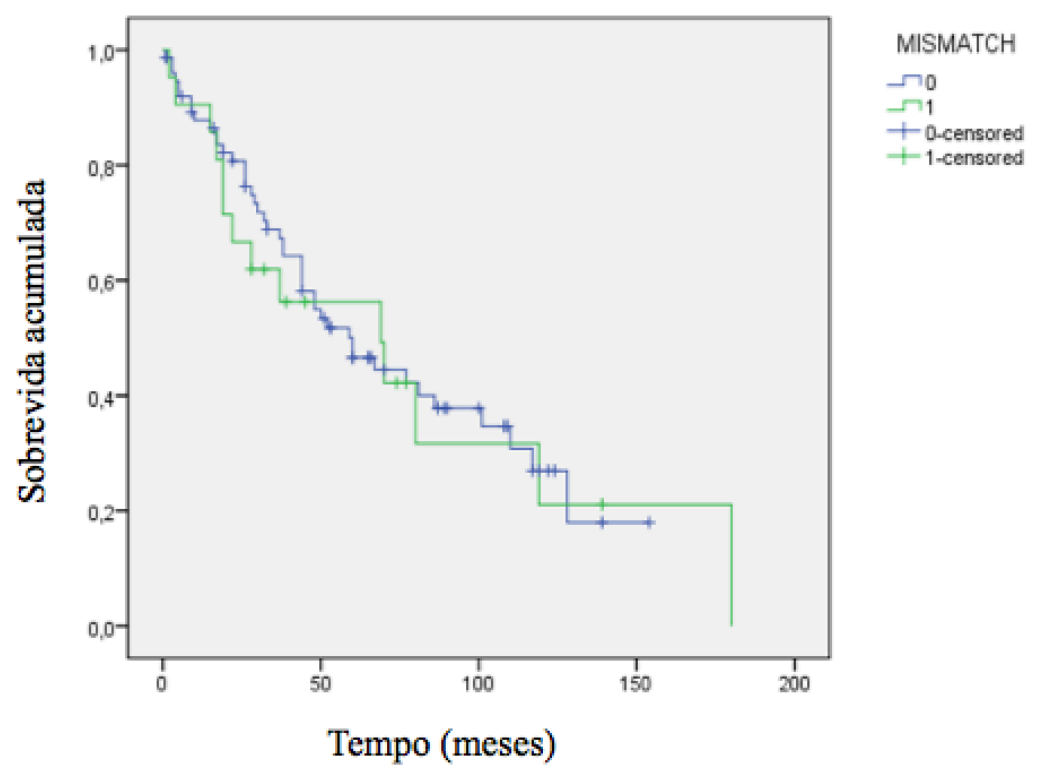

Figura 12: Estimativa de sobrevida pelo método Kaplan Meyer. Em azul: ausência do sinal Mismatch T2/FLAIR. Em verde: presenca do sinal.

\subsubsection{Entre os sexos masculino e feminino em relação a progressão de}

\section{doença:}

A análise estatística demonstrou não haver diferença na sobrevida em relação a progressão de doença nos sexos masculino e feminino $(p=0,12)$.

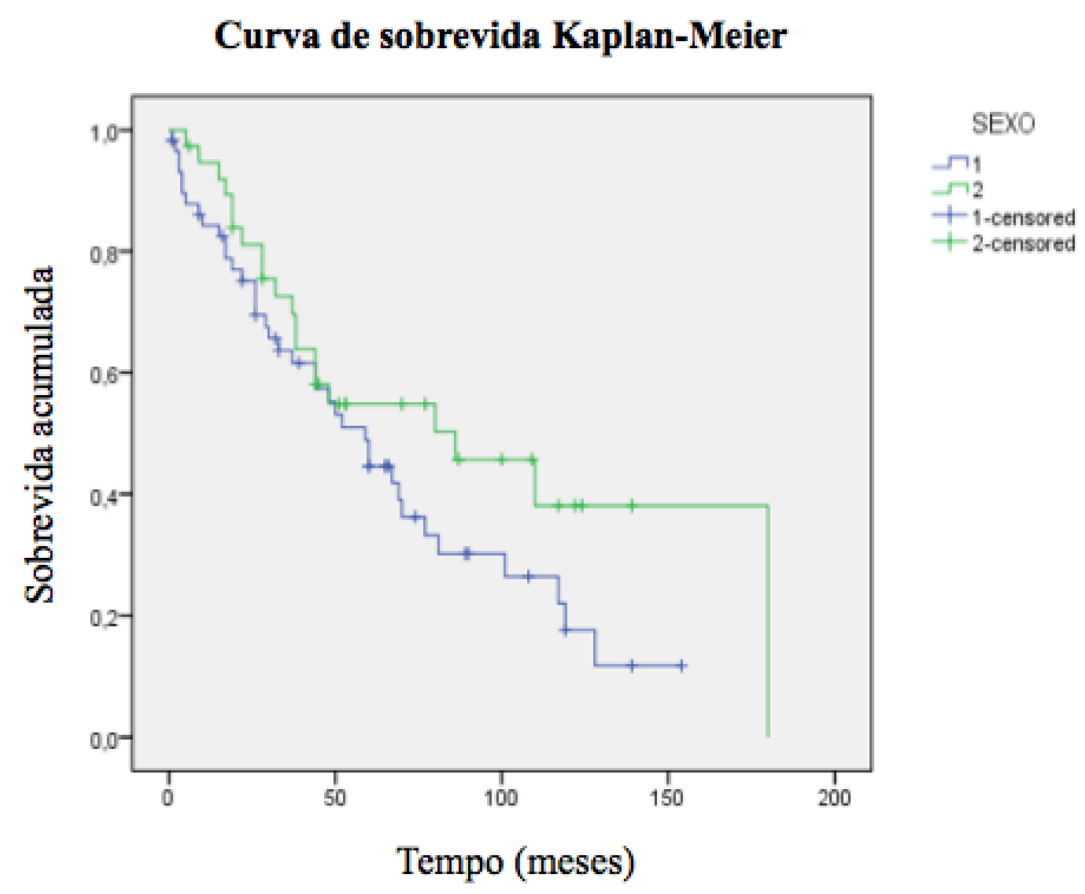

Figura 13: Estimativa de sobrevida pelo método Kaplan Meyer. Em azul: sexo masculino. Em verde: sexo feminino. 


\subsubsection{Comparação entre tipos histológicos em relação a} progressão de doença:

A análise estatística demonstrou não haver diferença na sobrevida em relação a progressão de doença entre os tipos histológicos $(p=0,086)$.

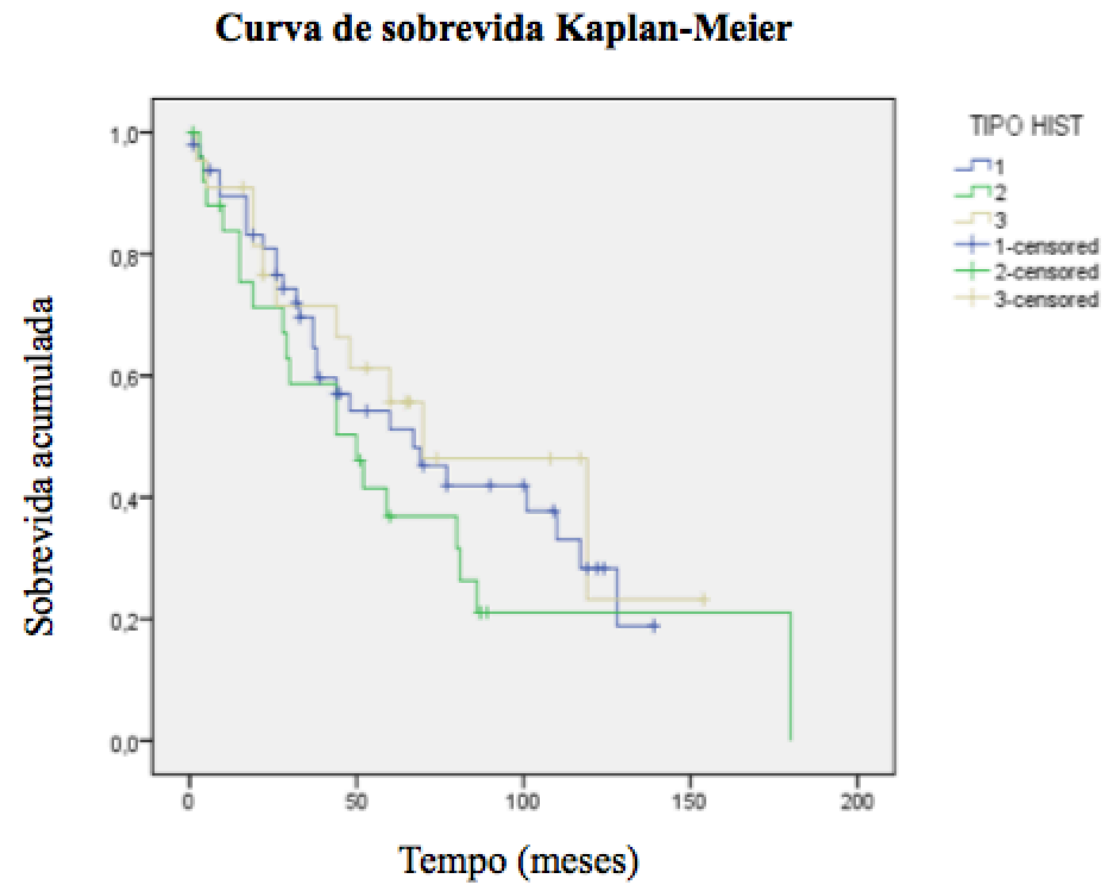

Figura 14: Estimativa de sobrevida pelo método Kaplan Meyer. Em azul: oligodendrogliomas. Em verde: oligoastrocitomas. Em amarelo: astrocitomas.

\subsubsection{Comparação entre os graus tumorais em relação a progressão de} doença:

A análise estatística demonstrou não haver diferença na sobrevida em relação a progressão de doença entre diferentes graus tumorais $(p=0,73)$. 


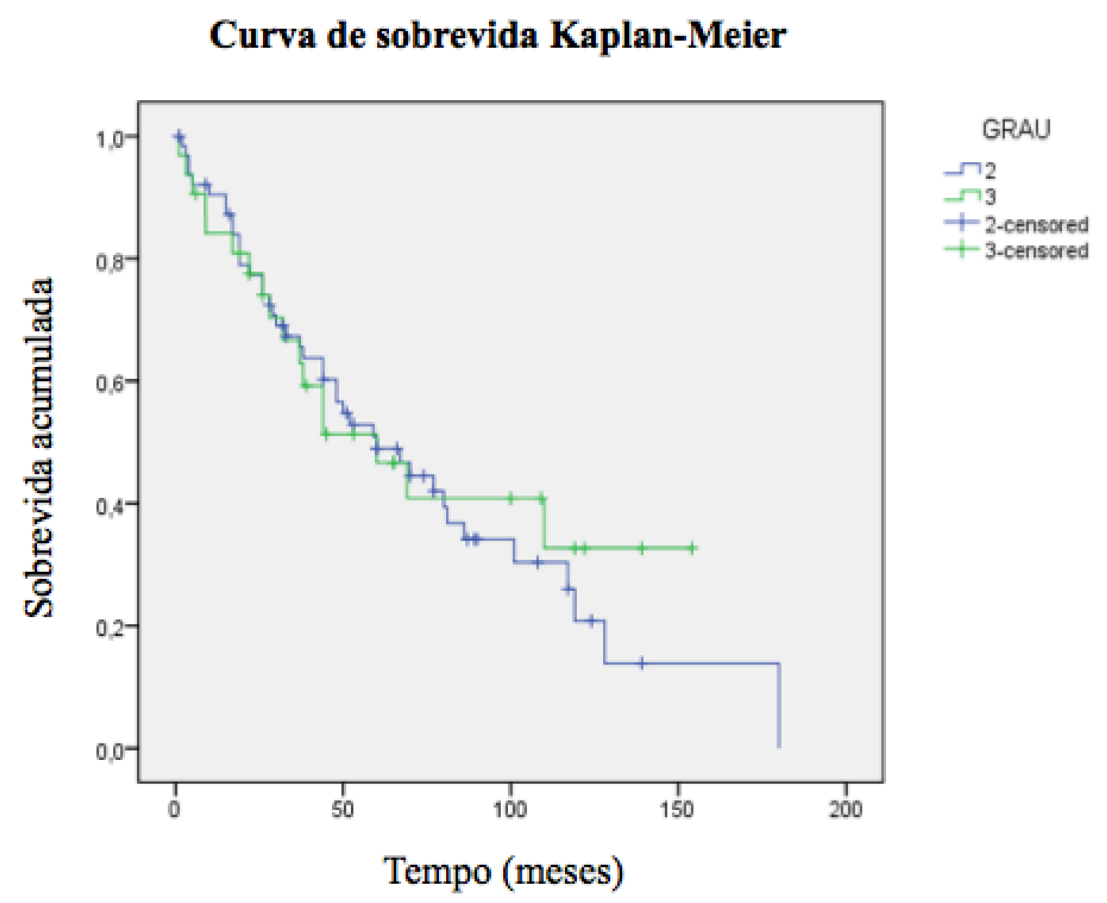

Figura 15: Estimativa de sobrevida pelo método Kaplan Meyer. Em azul: gliomas difusos grau II. Em verde: grau III.

4.2.5 Comparação entre as faixas etárias em relação à progressão de doença, considerando 40 anos como o divisor entre os grupos:

A análise estatística demonstrou não haver diferença na sobrevida em relação a progressão de doença entre os grupos acima e igual/abaixo de 40 anos $(p=0,73)$.

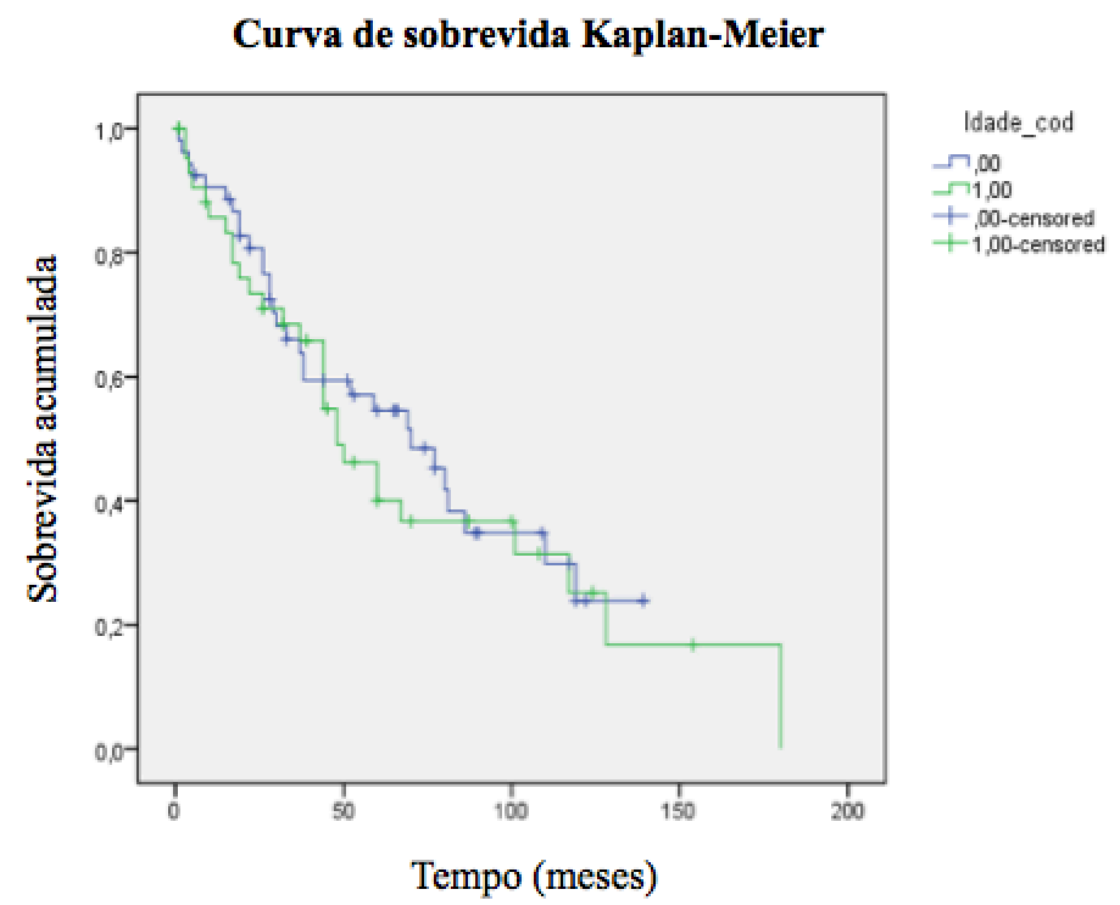

Figura 16: Estimativa de sobrevida pelo método Kaplan Meyer. Em azul: grupo com idade inferior a 40 anos. Em verde: idade superior . 1 in ...... 


\section{DISCUSSÃO}

O tratamento e manejo dos gliomas difusos passou por mudanças nas últimas décadas, com a introdução de novas tecnologias para auxiliar na abordagem cirúrgica, radioterápicas e quimioterápicas que melhoraram não só a sobrevida como a qualidade de vida dos pacientes. Neste contexto, a análise molecular dos tumores vem se consolidando como principal fator na classificação deste grupos de tumores, proporcionando informações prognósticas e probabilidade de resposta a tratamentos (10).

Neste cenário, o sinal Mismatch T2/FLAIR surgiu como um achado de imagem descrito recentemente na literatura, sendo estudado com o intuito principal de associar a imagem a um subtipo molecular específico de glioma difuso. Trata-se de fator relevante na avaliação deste grupo de tumores, notadamente em regiões onde a análise molecular ainda é pouco disponível, já que proporciona uma correlação altamente específica entre um marcador de imagem e um subtipo molecular tumoral.

A especificidade do sinal Mismatch T2-FLAIR foi de aproximadamente $100 \%$ segundo a descrição dos estudos de Patel et al e Broen et al, ou seja, em todos os casos com diagnóstico histopatológico de gliomas difusos nos quais o sinal de imagem estava presente, identificava-se o perfil molecular IDH mutado sem codelação 1p/19q, correspondendo, portanto, a astrocitomas. Johnson et al (15) descreveram recentemente um caso de paciente com diagnóstico de glioma difuso IDH mutado com codeleção 1p/19q (oligodendroglioma) que apresentava o sinal Mismatch T2-FLAIR no exame de imagem, demonstrando que, apesar de alta especificidade, a correlação entre a imagem e o perfil molecular pode apresentar exceções.

O sinal de imagem é de fácil acesso e avaliação no estudo pré-operatório, podendo contribuir para informações prognósticas, bem como para decisões terapêuticas. Sabe-se que os gliomas em geral beneficiam-se de ressecção tumoral total versus resecção subtotal, porém já é estabelecido que tumores sem codeleçao $1 p 19 q$ apresentam pior prognóstico e menor resposta a tratamento radio e quimioterápico. Nestes casos, ter a informação pré operatória de que o tumor não pertence a este grupo pode influenciar na decisão terapêutica do paciente de acordo com seu contexto clínico, como, por exemplo, se houver acometimento de áreas cerebrais eloquentes(15). 
Tentou-se, neste estudo, avaliar se a presença do sinal poderia, como fator isolado, ter relação com a sobrevida ou com a progressão de doença nos pacientes com gliomas difusos.

O estudo, no entanto, não demonstrou diferença significativa na sobrevida geral ou sobrevida sem progressão de doença entre os grupos de pacientes com presença ou ausência do sinal Mismatch T2/FLAIR na ressonância magnética pré-operatória. Se a presença do sinal sugere o diagnóstico de astrocitomas, e já é estabelecido que os astrocitomas apresentam pior prognóstico que os oligodendrogliomas, questiona-se o por que de não ter havido associação do sinal Mismatch T2-FLAIR com pior prognóstico. Uma das explicações possíveis é ausência da análise molecular disponível em grande parte dos pacientes avaliados, ou seja, não temos como ter certeza do diagnóstico completo e correto de cada um dos tumores, o que pode ter afetado a amostra em termos de comparação. Dos 21 pacientes que apresentaram o sinal Mismatch T2-FLAIR, 10 tinham o diagnóstico histológico de oligodrendrogliomas, o que é incompatível com a alta especificidade do sinal para o diagnóstico de astrocitomas. Ou seja, provavelmente estes 10 pacientes, ou pelo menos a grande maioria deles, corresponderiam a astrocitomas, mas a ausência de análise molecular impossibilita esta confirmação, o que constitui a grande limitação deste estudo.

Em relação às outras variáveis avaliadas e comparadas, como sexo, faixa etária e tipo histológico, também não houve diferença estatística significativa em relação a sobrevida.

Resultado estatisticamente significativo foi obtido em relação à sobrevida, independentemente de progressão ou não de doença, entre os grupos de tumores grau II e III (OMS), mostrando-se significativamente reduzida neste último grupo, aspecto já conhecido e consolidado na literatura e na prática clínico-radiológica (1). Em relação à progressão de doença comparando estes dois grupos, não houve diferença significativa.

O objetivo deste estudo não foi correlacionar a presença do sinal Mismatch T2/FLAIR com tipos moleculares de tumor, como realizado nos trabalhos de Patel et al e Broen et al. Não dispomos da análise molecular de grande parte dos pacientes avaliados. Estes trabalhos, como já mencionado, provaram estreita correlação da presença do sinal com um tipo molecular específico, não sendo avaliado o significado prognóstico isolado em relação a presença ou ausência do sinal.

Dos 10 pacientes nos quais a avaliação molecular estava disponível em nossa amostra, seis tumores apresentavam IDH mutado e codeleção 1p19q e quatro tumores apresentavem IDH mutado sem codeleção 1p19q. Neste grupo de dez pacientes, os dois casos nos quais o sinal Mismatch T2/FLAIR estava presente correspondiam a tumores 
sem codeleção 1p19q (astrocitomas), o que está de acordo com a alta especificidade do sinal descrita nos estudos de Patel et al e validada no estudo de Broen et al.

O significado do aspecto de imagem do sinal Mismatch T2/FLAIR permanece não esclarecido, sendo uma das hipóteses a relação com aumento de proteínas da via mTOR, achado que pode ser decorrente de mutações nesta via no curso dos tumores IDH mutados (5). Os aspectos foram pouco estudados e merecem mais investigação. Outra possibilidade a ser considerada é o fato do hipossinal FLAIR, considerando as características desta sequência de suprimir o sinal de conteúdo semelhante ao líquor, portanto líquido, representar um tumor de mais baixa celularidade, o que merece correlação histopatológica, aspecto não realizado neste trabalho.

Destacam-se algumas importantes limitações do estudo. Primeiramente, trata-se de um estudo retrospectivo, com limitações inerentes a este tipo de desenho. Além disso, a ausência da avaliação molecular na maioria dos tumores não permitiu a separação e comparação entre os grupos com e sem codeleção $1 p 19 q$, aspecto que é preconizado atualmente e mostra-se superior à simples análise histopatológica convencional. Outra limitação foi a análise do sinal Mismatch T2-FLAIR por apenas um médico radiologista. Algumas outras variáveis podem ter influenciado o resultado prognóstico do paciente, como grau de ressecção, envolvimento de áreas eloquentes, tratamento radioterápico e/ou quimioterápico.Por fim, quando foi utilizado óbito como desfecho, assumiu-se que a causa da morte foi relacionada ao tumor cerebral primário, o que pode não necessariamente ter ocorrido, tendo em vista que muitos dos pacientes tinham idade avançada, comorbidades e poderiam, portanto, ter o óbito relacionado a outras causas.

\section{CONCLUSÃO}

A presença do sinal de imagem Mismatch T2/FLAIR como fator isolado não teve relação com o prognóstico em pacientes com diagnóstico histopatológico de gliomas difusos.

Os tumores grau III apresentaram pior prognóstico em relação a sobrevida global quando comparados aos tumores grau II (OMS).

Não houve diferença significativa em relação a sobrevida global ou progressão de doença comparando variáveis como sexo, faixa etária e tipo histológico. 


\section{7 - REFERÊNCIAS:}

1 - Larsen, J., Wharton, S. B., McKevitt, F., Romanowski, C., Bridgewater, C., Zaki, H., \& Hoggard, N. (2017). "Low grade glioma": an update for radiologists. The British Journal of Radiology, 90(1070), 20160600.

2 - Chang Ming Zhang, and Daniel J. Brat. Genomic profiling of lower-grade gliomas uncovers cohesive disease groups: implications for diagnosis and treatment . Chin J Cancer. 2016 Jan 12;35:12.

3 - Louis DN, Perry A, Reifenberger G, et al. The 2016 World Health Organization Classification of Tumors of the Central Nervous System: a summary. Acta Neuropathol. 2016;131(6):803-20.

4 - Louis DN, Ohgaki H, Wiestler OD, Cavenee WK (2007) World Health Organization histological classification of tumours of the central nervous system. International Agency for Research on Cancer, Lyon.

5- Patel SH, Poisson LM, Brat DJ, et al. T2-FLAIR Mismatch, an Imaging Biomarker for IDH and $1 \mathrm{p} / 19 \mathrm{q}$ Status in Lower-grade Gliomas: A TCGA/TCIA Project. Clin Cancer Res. 2017;23(20):6078-85.

6 - Eckel-Passow JE, Lachance DH, Molinaro AM, Walsh KM, Decker PA, Sicotte H, et al. Glioma groups based on $1 \mathrm{p} / 19 \mathrm{q}$, IDH, and TERT promoter mutations in tumors. $N$ Engl $J$ Med 2015;372:2499-508.

7 - Broen MPG, Smits M, Wijnenga MMJ, Dubbink HJ, Anten MHME, Schijns OEMG, Beckervordersandforth J, Postma AA, van den Bent MJ. The T2-FLAIR mismatch sign as an imaging marker for non-enhancing IDH-mutant, 1p/19q-intact lower-grade glioma: a validation study. Neuro Oncol. 2018 Sep 3;20(10):1393-1399.

8 - Cancer Genome Atlas Research Network, Brat DJ, Verhaak RG, Aldape KD, Yung WK, Salama SR, Cooper LA, Rheinbay E, Miller CR, Vitucci M et al (2015) Comprehensive, integrative genomic analysis of diffuse lower-grade gliomas. $N$ Engl J Med 372:2481-2498.

9 - van den Bent MJ, Brandes AA, Taphoorn MJ, et al. Adjuvant procarbazine, lomustine, and vincristine chemotherapy in newly diagnosed anaplastic oligodendroglioma: long-term followup of EORTC brain tumor group study 26951. J Clin Oncol. 2013; 31:344-50.

10- A. Hilario, J.M. Sepulveda, A. Perez-Nuñ ez, E. Salvador, J.M. Millan, A. Hernandez-Lain, V. Rodriguez-Gonzalez, A. Lagares, and A. Ramos Prognostic Model Based on Preoperative MRI Predicts Overall Survival in Patients with Diffuse Gliomas

11 -Jansen M, Yip S, Louis DN. Molecular pathology in adult gliomas: diagnostic, prognostic and predictive markers. Lancet Neurol 2010;9:717-26

12 - HardestyDA, SanaiN.The value of glioma extent ofr esection in the modern neurosurgical era. Front Neurol 2012;3:140 - 47.

13 - Kawaguchi T, Sonoda Y, Shibahara I, Saito R, Kanamori M, Kumabe T, et al. Impact of gross total resection in patients with WHO grade III glioma harboring the IDH $1 / 2$ mutation without the 1p/19q co-deletion. Journal of neuro-oncology. 2016;129:505-14.

14: Osborn's Brain: Imaging, Pathology, and Anatomy. By Anne G. Osborn Lippincott, Williams \& Wilkins, Philadelphia, PA and Amirsys Publishing: Salt Lake City, UT. 2012. 
15: Johnson DR, Kaufmann TJ, Patel SH, Chi AS, Snuderl M, Jain R There is an exception to every rule-T2-FLAIR mismatch sign in gliomas. Neuroradiology 2019 Feb;61(2):225-227. 